\title{
CONDITIONS PROVIDING OPTIMUM SEPARATION
}

\author{
EUGENE BARSKY* \\ The Department of Industrial Engineering and Management, \\ Sami Shamoon College of Engineering, Beer-Sheva, Israel
}

(Received 12 October 2004; Accepted 19 October 2004)

\begin{abstract}
It is generally accepted in the field of powder technology that the most objective quality criterion for the optimization of separation is the Hancock criterion. In this article, use an analytical procedure to show disadvantages of this criterion. I also apply the same analysis to show the objectivity of the entropy criterion for the optimization of separation processes. A simple objective relationship for the optimization of separation processes of pourable materials is derived on the basis of the entropy criterion.
\end{abstract}

Keywords: Entropy; Separation; Criterion; Optimization

\section{INTRODUCTION}

Regardless of the technology used, the purpose of a separator is always the same: to partition the original material into two products along a certain size boundary so as to obtain the greatest possible extent of separation.

Let one consider for the general case the phenomena that unfold when such a separation is carried out. One shall assume that a certain pourable material has an initial composition such that the grain-size distribution of the successive residues may be represented by the curve $\mathrm{ABC}$ in Fig. 1. Let one say that for technical reasons it is necessary to separate this material along the $x_{0}$ micrometer boundary. On a certain scale the area bounded by the curve $\mathrm{ABC}$ and the coordinate axis will correspond to the total quantity of material at the input. Let one arbitrarily take this quantity to be unity and let one designate this curve by $Q(x)$.

In the ideal case the separation will proceed along $B x_{0}$. This line divides the initial composition into two parts:

$D_{s}$ the fine product

$R_{s}$ the coarse product

*E-mail: eugeneb@bgumail.bgu.ac.il 


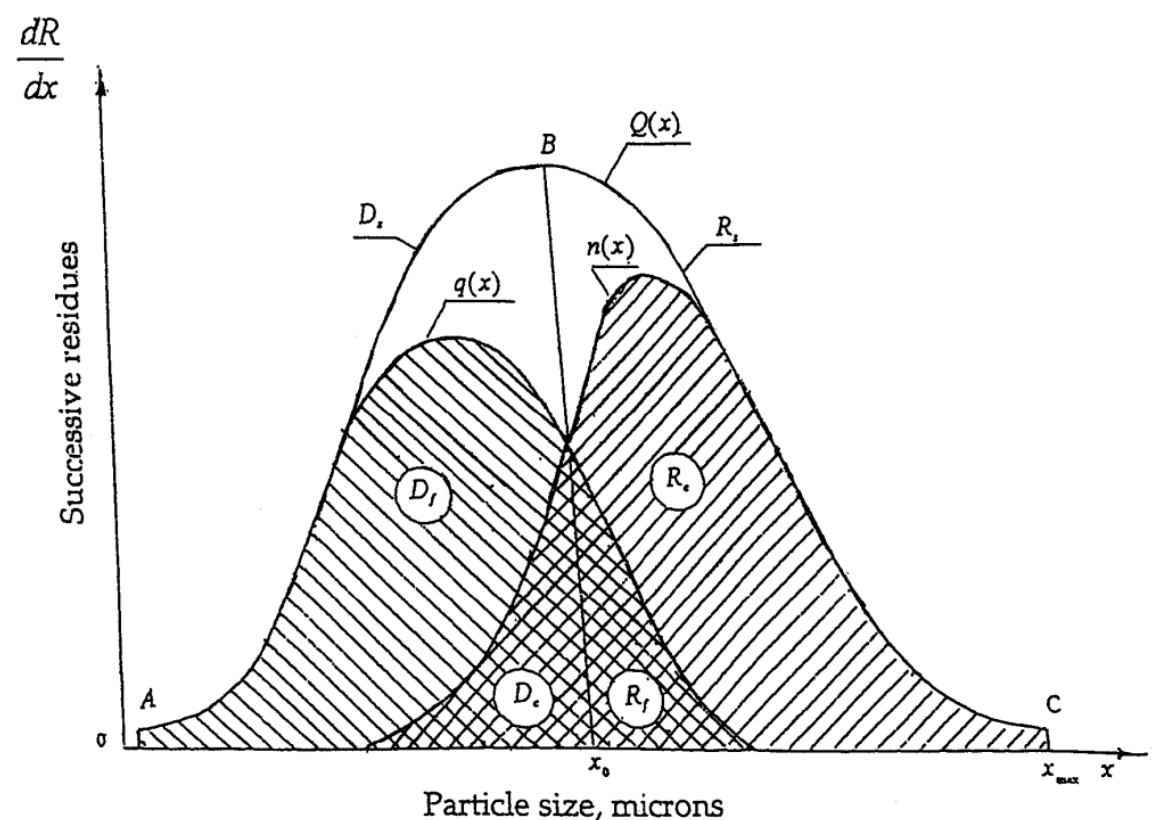

FIGURE 1 Grain-size distribution of successive residues in the separation of a pourable material.

In reality, the process of separation does not proceed ideally since some of the fine fractions find their way into the coarse product and some of the coarse fractions into the fines.

Let the fine product in the real process be described by the curve $q(x)$ and the coarse product by the curve $n(x)$ in these coordinates. From the way in which these curves were obtained it is evident that at any point $x_{i}$ the following relation will hold for fraction $x_{i}$ :

$$
Q\left(x_{i}\right)=q\left(x_{i}\right)+n\left(x_{i}\right)
$$

for the following extent of fractional separation:

$$
F_{f}\left(x_{i}\right)+F_{c}\left(x_{i}\right)=1
$$

where

$$
F_{f}\left(x_{i}\right)=\frac{q\left(x_{i}\right)}{Q\left(x_{i}\right)}
$$

is the entrainment of the narrow class in the fine product and

$$
F_{c}\left(x_{i}\right)=\frac{n\left(x_{i}\right)}{Q\left(x_{i}\right)}
$$


is the entrainment of the narrow class into the coarse product, $Q(x)$ is the amount of narrow class in the input, and $q\left(x_{i}\right)$ and $n\left(x_{i}\right)$ are, respectively, the amounts of narrow class in the fine and in the coarse product.

\section{ANALYSIS OF EXISTING METHODS}

There are a number of approaches for the direct determination of the conditions that will assure an optimum process (without calculating the efficacy). These approaches relate the quality of the separation process to the size of the 'boundary grain size', a concept with a well-defined physical meaning. This meaning is that for any given relation between the regime and design parameters, it is always possible to choose a narrow size class - or boundary grain size - for which the given process will be optimal.

Rubinchik [1] determines the limiting grain size using relations for an ideal process and proposes that the optimal efficacy be determined based on the requirement that the fine product (as percentage of total output) be equal to the content (percentage) of the fines in the initial material:

$$
D_{s}=\gamma_{f}
$$

where $\gamma_{f}$ is the fine product output.

Bond [2] attempts to find the boundary grain size by taking the content of fines in the fine product that is equal to the content of coarse particles in the coarse product:

$$
\frac{D_{f}}{\gamma_{f}}=\frac{R_{c}}{\gamma_{c}}
$$

where $\gamma_{c}$ is the coarse product output.

Povarov [3] suggests finding the required boundary grain size by taking the particular narrow size class whose relative content in the initial material and in both separation products is the same:

$$
Q(x)=\frac{q(x)}{X_{f}}=\frac{n(x)}{X_{c}}
$$

Steinmetzer [4] suggests taking the size of the boundary grain size to be the narrow size class which yields two products with the same proportion $(50 \%)$ of particles of that size:

$$
F_{f}(x)=F_{c}(x)
$$

None of these methods has been proven and their validity is still being debated [5].

Let us try to analyse the question by examining the essence of the problems that separation sets out to solve.

In the general case, the area of the graph in Fig. 1 is cut into four parts by the lines of the ideal and real process: $D_{f}$-fine material at the fine product outlet; $R_{f}$-coarse material at the fine product outlet; $R_{c}$-coarse material at the coarse product outlet; $D_{c}$-fine material at the coarse product outlet. 
For all these parts the following relations obviously hold:

$$
\begin{aligned}
& D_{s}+R_{s}=1 \\
& D_{f}+D_{c}=D_{s} \\
& R_{c}+R_{f}=R_{s} \\
& R_{f}+D_{f}=\gamma_{f} \\
& R_{c}+D_{c}=\gamma_{c}
\end{aligned}
$$

Some simple characteristics are usually formulated based on the above parameters:

$$
\begin{aligned}
\varepsilon_{f} & =\frac{D_{f}}{D_{s}}-\text { fines entrainment } \\
\varepsilon_{c} & =\frac{R_{c}}{R_{s}}-\text { coarse particle entrainment } \\
k_{f} & =\frac{R_{f}}{R_{s}}-\text { fines contamination } \\
k_{c} & =\frac{D_{c}}{D_{s}}-\text { coarse particle contamination }
\end{aligned}
$$

To find the conditions assuring maximum fractional distinction between the separation products, it is necessary to maximize the following relation:

$$
E_{\mathrm{I}}=D_{f}+R_{c}
$$

or, which amounts to the same, to minimize:

$$
E_{\mathrm{II}}=R_{f}+D_{c}
$$

The optimization conditions, in accordance with these relations, are given by

$$
\frac{d E_{\mathrm{I}}}{d x}=0 ; \quad \frac{d E_{\mathrm{II}}}{d x}=0
$$

It is easy to show that both relations give the same result. Let us expand them:

$$
\frac{d E_{\mathrm{I}}}{d x}=\frac{d D_{f}}{d x}+\frac{d R_{c}}{d x}=\frac{d \int_{0}^{x} q(x) d x}{d x}+\frac{d \int_{x}^{x_{\max }} n(x) d x}{d x}=0
$$

It is known that the derivative of a certain integral with variable upper limit and constant lower limit is equal to the expression under the integral sign at the point of the upper limit. Hence

$$
\begin{aligned}
& q(x)-n(x)=0, \quad \text { i.e. } \\
& q(x)=n(x)
\end{aligned}
$$


It follows from Eq. (1) that, at the optimum point, both products - fine and coarsecontain the same relative amount of the narrow size class, i.e. the optimality conditions correspond to:

$$
F_{f}=F_{s}=0.5
$$

Only one point in Fig. 1 meets this condition - the intersection of the curves $g(x)$ and $n(x)$. This point corresponds to the $x_{0}$ ordinate.

Thus for any separation process one can always find the boundary size that corresponds to the optimal result. For the first time the validity of relation (14) has been rigorously established.

However, while this parameter is necessary in order to determine the process optimum, it is clearly not sufficient. Indeed, the values of $D_{c}$ and $R_{f}$ may themselves be greater or smaller, but they do not define the degree of completeness of the process in a numerical expression. Many criteria have been proposed for evaluating this degree of completeness [5].

\section{THE NEW METHOD OF OPTIMIZATION}

For assessment of whether these criteria fit the problems that are solvable by the separation process, the following conditions were imposed:

(1) The criterion should be monotonous, i.e., it should have the largest value for the separation process that provides the largest fractional difference.

(2) The criterion should have zero value upon fractionation of the material without affecting the fractional composition or upon use of all the material for the production of a single product.

(3) The criterion should have the maximum value in an ideal separation.

(4) The criterion should be defined well, i.e., not dependent on the product according to which the criterion is calculated.

Analysis of many criteria shows that the following two criteria best meet the requirements of separation:

(1) Hancock criterion [5]

For the fine product:

$$
E_{f}=\frac{D_{f}}{D_{s}}-\frac{R_{f}}{R_{s}}
$$

For the coarse product:

$$
E_{c}=\frac{R_{c}}{R_{s}}-\frac{D_{c}}{D_{s}}
$$

(2) Entropy criterion [6]

$$
\begin{aligned}
E= & -\left\{\left[D_{s} \ln D_{s}+R_{s} \ln R_{s}\right]-\gamma_{f}\left[D_{f}^{\prime} \ln D_{f}^{\prime}+R_{f}^{\prime} \ln R_{f}^{\prime}\right] .\right. \\
& \left.-\gamma_{c}\left[R_{c}^{\prime} \ln R_{c}^{\prime}+D_{c}^{\prime} \ln D_{c}^{\prime}\right]\right\}
\end{aligned}
$$


where $D_{f}^{\prime}, R_{f}^{\prime}, D_{c}^{\prime}$ and $R_{c}^{\prime}$ are parameters referring to the product yield. Equation (17) makes use of the following relations:

$$
\begin{aligned}
& R_{f}^{\prime}=\frac{R_{f}}{\gamma_{f}} ; \quad D_{f}^{\prime}=\frac{D_{f}}{\gamma_{f}} ; \\
& R_{c}^{\prime}=\frac{R_{c}}{\gamma_{c}} ; \quad D_{c}^{\prime}=\frac{D_{c}}{\gamma_{c}} ; \\
& R_{f}^{\prime}+D_{f}^{\prime}=1 ; \quad R_{c}^{\prime}+D_{c}^{\prime}=1
\end{aligned}
$$

Hancock's criterion seems to fit the above conditions:

(1) It is monotonous, as has been shown for over 100 years of use.

(2) Upon separation of the material without changing the fractional composition: $D_{f}=m D_{s}, R_{f}=m R_{s}$, the following will be obtained:

$$
E_{f}=\frac{D_{f}}{D_{s}}-\frac{R_{f}}{R_{s}}=\frac{m D_{s}}{D_{s}}-\frac{m R_{s}}{R_{s}}=m-m=0
$$

(3) In the case of ideal separation:

$$
\begin{aligned}
& D_{f}=D_{s}, \quad R_{f}=0 \\
& E_{f}=\frac{D_{f}}{D_{s}}-\frac{R_{f}}{R_{s}}=\frac{D_{s}}{D_{s}}-\frac{0}{R_{s}}=1
\end{aligned}
$$

(4) It does not matter for what product the value of the criterion is calculated; this means that the criterion has been properly defined.

$$
\begin{aligned}
E_{f}-E_{c} & =\frac{D_{f}}{D_{s}}-\frac{R_{f}}{R_{s}}-\frac{R c}{R_{s}}+\frac{D_{c}}{D_{s}}=\frac{D_{f}+D_{c}}{D_{s}}-\frac{R_{f}+R_{c}}{R_{s}} \\
& =\frac{D_{s}}{D_{s}}-\frac{R_{s}}{R_{s}}=11=0
\end{aligned}
$$

It is interesting to note that parameter (15) is universally recognized and is widely used in both the specialized literature and in the field. As to the criterion (17), it has been proposed relatively recently and therefore is not widely known.

Using relations (13) and (14), let us try to analyse these criteria from the standpoint of correspondence to optimal conditions. The condition of optimality is given by

$$
\frac{\mathrm{d} E}{\mathrm{~d} X}=0
$$

Let one begin by expanding this condition for the dependence given by Eq. (17). We can write:

$$
E=-\left[\left(R_{s} \ln R_{s}+D_{s} \ln D_{s}\right)-\left(R_{c} \ln \frac{R}{\gamma_{c}}+D_{c} \ln \frac{D_{c}}{\gamma_{c}}+D_{f} \ln \frac{D_{f}}{\gamma_{f}}+R_{f} \ln \frac{R_{f}}{\gamma_{f}}\right)\right]
$$


After several transformations the derivative of Eq. (24) becomes

$$
\begin{aligned}
\frac{d E}{d x}= & Q(x) \ln R_{s}-Q(x) \ln D_{s}-n(x) \ln \frac{R_{c}}{\gamma_{c}}+n(x) \ln \frac{D_{c}}{\gamma_{c}} \\
& +q(x) \ln \frac{D_{f}}{\gamma_{f}}-q(x) \ln \frac{R_{f}}{\gamma_{f}}=0
\end{aligned}
$$

Hence

$$
Q(x)\left[\ln R_{s}-\ln D_{s}\right]=n(x)\left[\ln \frac{R_{c}}{\gamma_{c}}-\ln \frac{D_{c}}{\gamma_{c}}\right]+q(x)\left[\ln \frac{R_{f}}{\gamma_{f}}-\ln \frac{D_{f}}{\gamma_{f}}\right]
$$

or

$$
\ln \frac{R_{s}}{D_{s}}=F_{c}(x) \ln \frac{R_{c}}{D_{c}}+F_{f}(x) \ln \frac{R_{f}}{D_{f}}
$$

Let us carry out a few transformations:

$$
\ln \frac{R_{s}}{D_{s}}-\ln \frac{R_{c}}{D_{c}}=F_{f}(x)\left[\ln \frac{R_{f}}{D_{f}}-\ln \frac{R_{c}}{D_{c}}\right]
$$

whence

$$
\ln \left(\frac{R_{s}}{D_{s}} \cdot \frac{D_{c}}{R_{c}}\right)=F_{f}(x) \ln \frac{R_{f} \cdot D_{c}}{R_{c} \cdot D_{f}}
$$

From Eq. (8), we can write

$$
\ln \frac{k_{c}}{\varepsilon_{c}}=F_{f}(x) \ln \frac{k_{f} \cdot k_{c}}{\varepsilon_{c} \cdot \varepsilon_{f}}
$$

Bearing in mind that in an optimal regime $F_{f}(x)=0.5$, it follows that:

$$
\frac{k_{c}}{\varepsilon_{c}}=\sqrt{\frac{k_{f} \cdot k_{c}}{\varepsilon_{f} \cdot \varepsilon_{c}}}
$$

Equation (3) can hold only in one case, that is if

$$
\varepsilon_{f}=\varepsilon_{c}
$$

From Eq. (8) this automatically equals

$$
k_{c}=k_{f},
$$

as well, since in an optimal regime $0.5 \leq \varepsilon_{f}$ and $\varepsilon_{c} \leq 1$. 


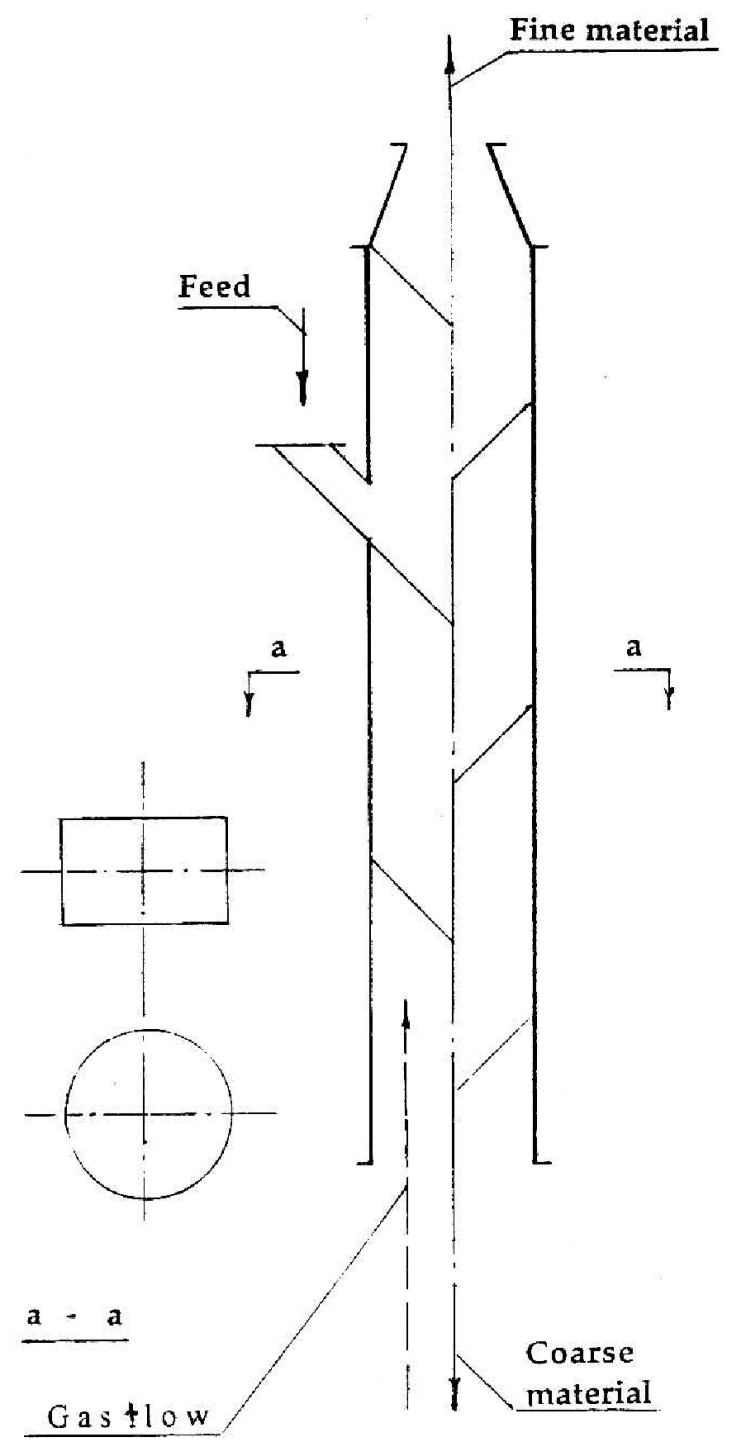

FIGURE 2 Schematic diagram of a cascade classifier.

Thus, analysis of the optimality conditions for the fairly complex entropy criterion gives us a simple dependence (32) expressing a simple condition of optimality for the process of separation. The cascade classifier containing stages is presented in Fig. 2. It consists of a vertical tube with a rectangular cross section. Alternating elements, representing the stages of the separator, are present inside the tube. The material is poured into the tube from above. The fine fraction leaves the classifier from the upper outlet, and the coarse fraction comes out from the lower outlet, against the air flow. Separation of ground quartzite in such an apparatus was performed at an airflow speed of $w=1.83 \mathrm{~m} / \mathrm{s}$. 


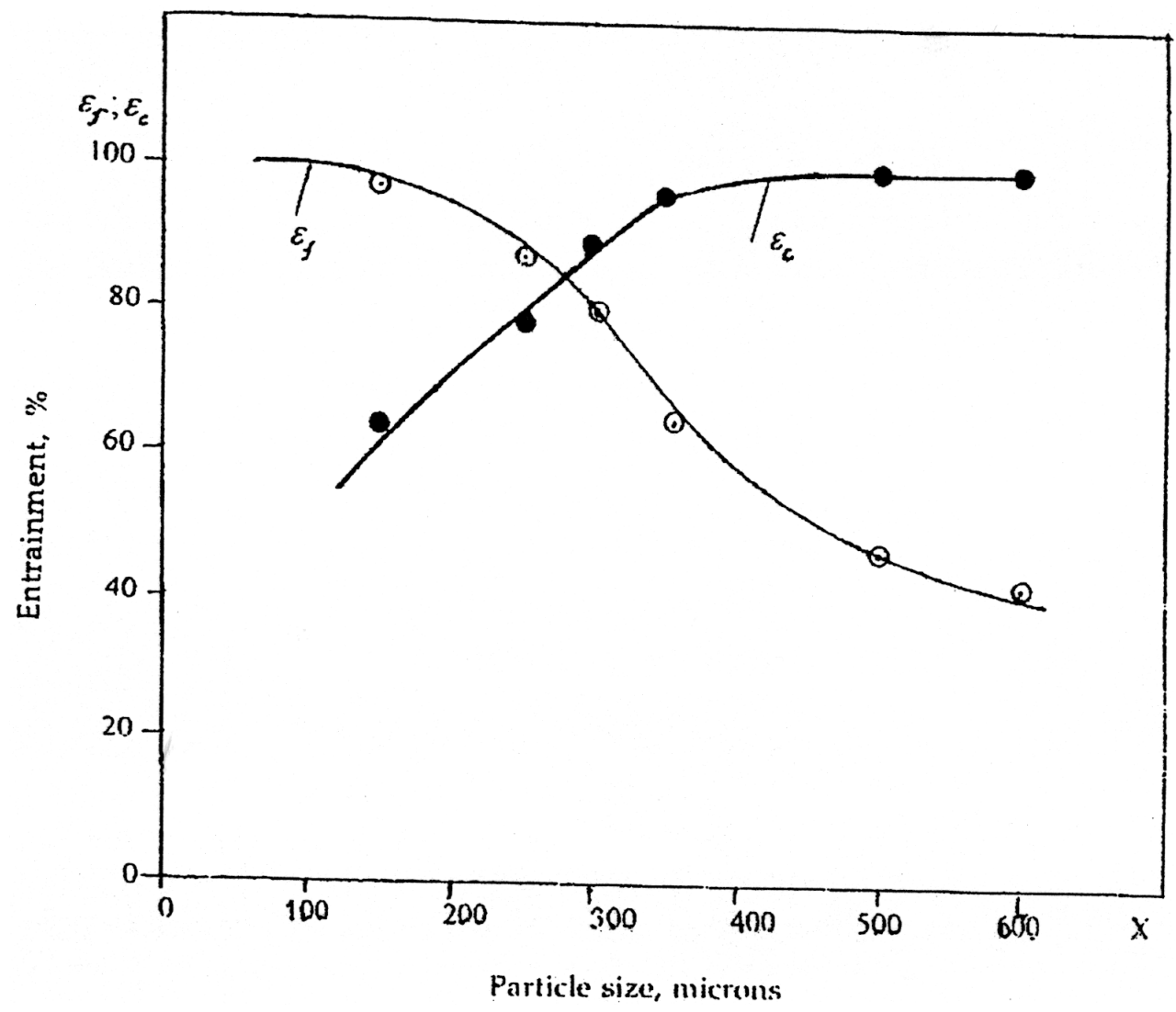

FIGURE 3 Entrainment of fine and coarse products as a function of the boundary grain size for a cascading classifier with air flow speed of $1.5 \mathrm{~m} \mathrm{sec}^{-1}$.

Figure 3 shows how criterion (31) assists in the determination the optimal grain size at which the separation is carried out. The grain size was $275 \mu \mathrm{m}$ and the yield was $84 \%$. Optimal parameters of other classifiers can be determined in a similar manner.

Now let one look at the optimality conditions for the Hancock criterion (14):

$$
\frac{d F_{f}}{d x}=0 ; \quad \frac{d F_{c}}{d x}=0
$$

Let us expand the first of these:

$$
\frac{q(x) \cdot D_{s}-D_{f} Q(x)}{D_{s}^{2}}-\frac{-q(x) R_{s}+Q(x) R_{f}}{R_{s}^{2}}=0
$$

From this

$$
\frac{F_{f}(x)}{D_{s}}-\frac{\varepsilon_{f}}{D_{s}}=\frac{k_{f}}{R_{s}}-\frac{F_{f}(x)}{R_{s}}
$$


From the above

$$
F_{f}(x)=k_{f} D_{s}+\varepsilon_{f} R_{s}=R_{s}\left(\varepsilon_{f}-k_{f}\right)+k_{f}
$$

Noting that the brackets contain the expression for the efficacy (8), one can write

$$
F_{f}(x)=E_{f} \cdot R_{s}+k_{f}
$$

Similarly for $F_{c}$ we obtain

$$
F_{c}(x)=E_{c} D_{s}+k_{c}
$$

From the above one can find the relations for the efficacy:

$$
\begin{gathered}
E_{f}=\frac{F_{f}(x)-k_{f}}{R_{s}} \\
E_{c}=\frac{F_{c}(x)-k_{c}}{D_{s}}
\end{gathered}
$$

It is always true that $E_{f}=E_{c}$, and therefore:

$$
\frac{F_{f}(x)-k_{f}}{R_{s}}=\frac{F_{c}(x)-k_{c}}{D_{s}}
$$

We found that under optimal conditions

$$
F_{f}(x)=F_{c}(x) \text { and } k_{f}=k_{c}
$$

In view of the above, Eq. (41) can only be valid in the particular case where

$$
D_{s}=R_{s}=0.5
$$

In all other cases the Hancock criterion cannot be used to optimize processes of separation.

\section{CONCLUSION}

Thus, my analysis has resulted in the formulation and demonstration of new optimality conditions for the separation of pourable materials. These conditions are relatively simple and convenient to use, as well as in the refutation of a numerical criterion which has been widely used to optimize such processes. 


\section{References}

[1] V.A. Olevskii, Construction and Calculation of Mechanical Classifiers and Hydrocyclones, Gosgortekhizdat, Moscow (1960), p. 314 (In Russian).

[2] F. Bond, Theory of Isotope Separation, 4 (1960), 18-36.

[3] F.I. Povarov, Technological evaluation of the functioning of classifiers. Obogashchenie Rud, 5 (1956), 40-48 (In Russian).

[4] S. Steinmetzer, Windsichtung der Feinhonle, Berlin, 1941, p. 326.

[5] M.D. Barsky, Optimization of Processes of Separation of Granular Materials, Nedra, Moscow (1978), p. 167 (In Russian).

[6] M. Barsky and E. Barsky, Criterion for separation of pourable material into $n$ components. In: Proc. 29th Int. Symp. Computer Appl. Miner. Industr., Beijing, China, 2001. 